\title{
Mapping the local nanostructure inside a specimen by tomographic small-angle $x$-ray scattering
}

\author{
C. G. Schroer, ${ }^{a)}$ M. Kuhlmann, S. V. Roth, and R. Gehrke \\ HASYLAB at DESY, Notkestrasse 85, D-22607 Hamburg, Germany \\ N. Stribeck and A. Almendarez-Camarillo \\ Institute of Technical and Molecular Chemistry, Hamburg University, Bundesstrasse 45, D-20146 Hamburg, \\ Germany \\ B. Lengeler \\ II. Physikalisches Institut, Aachen University, D-52056 Aachen, Germany
}

(Received 23 November 2005; accepted 7 March 2006; published online 18 April 2006)

\begin{abstract}
Small-angle $\mathrm{x}$-ray scattering is combined with scanning microtomography to reconstruct the small-angle diffraction pattern in the direction of the tomographic rotation axis at each location on a virtual section through a specimen. These data yield information about the local nanoscale structure of the sample. With rotational symmetry present in the diffraction patterns, e.g., for isotropic or fiber-textured scatterers, the full reciprocal space information in the small-angle scattering regime can be reconstructed at each location inside the specimen. The method is illustrated investigating a polymer rod made by injection molding. (C) 2006 American Institute of Physics. [DOI: 10.1063/1.2196062]
\end{abstract}

Hard x-ray scanning microscopy allows one to perform $\mathrm{x}$-ray analytical techniques, such as fluorescence analysis, absorption spectroscopy, and diffraction with high spatial resolution, revealing the elemental composition, the chemical state, and the nanostructure of a specimen. In combination with tomographic techniques, the structure inside a specimen can be reconstructed without the need for destructive sample preparation. A variety of hard x-ray microtomography techniques has been developed in the past few years, such as fluorescence microtomography ${ }^{1}$ and tomographic $\mathrm{x}$-ray absorption spectroscopy. ${ }^{2}$ The main advantage of these techniques is that one can obtain elemental and chemical information from inside a specimen.

In this letter we exploit small-angle x-ray scattering (SAXS) as contrast in scanning microtomography to obtain information about the local nanostructure on a virtual section through a sample. A variety of scanning micro-SAXS techniques has been developed over the past few years and is widely used to determine the local nanostructure in many fields of science and technology. ${ }^{3}$ SAXS contrast is of particular interest for samples that are homogeneous in elemental composition and density, but whose nanoscopic structure varies as a function of position. As an example, we investigate the variation in structure resulting from the solidification of polyethylene inside a mold during injection molding. While the density and composition of the polyethylene rod are quite homogeneous, its nanoscopic structure and thus its mechanical properties are not.

The experimental setup is shown in Fig. 1. Monochromatic $x$ rays coming from the left are focused onto the sample position by means of an appropriate x-ray optic. The guard slit suppresses parasitic scattering from optical components and residual air paths upstream. The small-angle diffraction pattern is recorded by a two-dimensional (2D) de-

\footnotetext{
${ }^{a)}$ Present address: Institute for Structural Physics, TU Dresden, D-01062
} Dresden, Germany; electronic mail: schroer@xray-lens.de tector placed at a distance $L_{\mathrm{SD}}$ behind the sample. Primary and transmitted intensities are measured by an ionization chamber and a positive-intrinsic-negative (PIN) diode inside a lead beam stop, respectively. The sample is mounted on a stage with three translations $(s, r$, and $z)[$ Fig. 1(b)] and one rotation $\phi$ about the vertical $z$ axis. The coordinate system $(x, y, z)$ is fixed in the sample frame.

Prior to a tomographic scan, the position of the virtual slice inside the sample is fixed using the $z$ translation. The $s$ translation along the beam is positioned such as to bring the axis of rotation into the focus. To record a SAXS tomogram, the sample is scanned through the microbeam in translation along $r$ and rotation $\phi$. To acquire a single tomographic projection, the sample is scanned through the microbeam in translation along $r$, recording at each position of the scan a full SAXS pattern with the 2D detector and the incident $\left(I_{0}\right)$ and transmitted $\left(I_{1}\right)$ fluxes with the ionization chamber and the PIN diode, respectively. After a projection is completed,

(a) side view

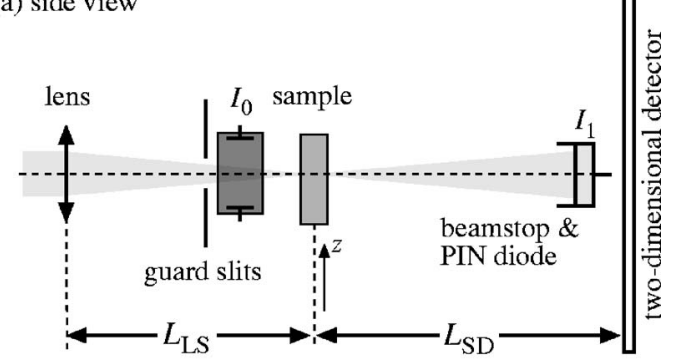

(b) top view of

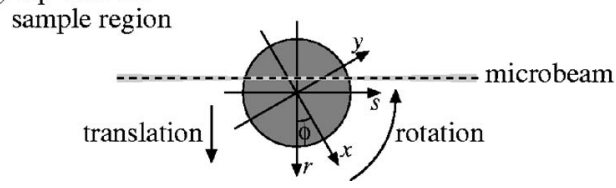

FIG. 1. (a) Side view of the setup for micro-small-angle scattering tomography. (b) Top view of the sample region. 

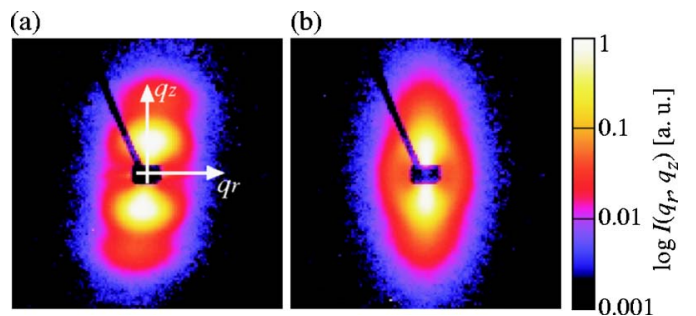

FIG. 2. (Color online) Small-angle x-ray scattering patterns of an injectionmolded polyethylene rod (cylinder axis parallel to $z$ ) recorded with an x-ray microbeam impinging (a) peripherally and (b) centrally. The intensity scale is logarithmic.

the sample is rotated by an integer fraction of $180^{\circ}$ and the next projection is recorded. This procedure is repeated until the sample has completed a $180^{\circ}$ rotation.

We have acquired a SAXS tomogram of a polyethylene rod (Lupolen 6021D by BASF) that was made by injection molding ${ }^{4}$ and that was subsequently stretched at elevated temperatures $\left(T \approx 80{ }^{\circ} \mathrm{C}\right)$. To this end, the $\mathrm{x}$ rays from the wiggler source at beamline BW4 at HASYLAB were monochromatized by a $\mathrm{Si}$ (111) double crystal monochromator $(\lambda=1.38 \AA)$. The first pair of slits at $20.2 \mathrm{~m}$ from the source was used to define a secondary source that was imaged onto the sample position by parabolic refractive $\mathrm{x}$-ray lenses made of beryllium. ${ }^{5}$ The resulting beam on the sample had a lateral full width at half maximum size of $57 \times 39 \mu \mathrm{m}^{2}$ (horizontal $\times$ vertical), a divergence of approximately $0.2 \mathrm{mrad}$, and a flux of the order of $10^{8}-10^{9} \mathrm{ph} / \mathrm{s}$. A 2D detector (MARCCD 165 by Mar Research) was placed at a distance of $L_{\mathrm{SD}}=1850 \mathrm{~mm}$ behind the sample, covering a $q$ range from 0.06 to $2 \mathrm{~nm}^{-1}$. 101 projections with 69 translational steps of $\Delta r \approx 80 \mu \mathrm{m}$ were recorded. At each position in the scan, the exposure time was $20 \mathrm{~s}$. Figure 2 shows two SAXS patterns recorded with the microbeam impinging peripherally and centrally onto the sample.

The attenuation coefficient $\mu(x, y)$ can be reconstructed from $I_{1}=I_{0} \exp \left\{-\int d s^{\prime} \mu\left[x\left(s^{\prime}, r\right), y\left(s^{\prime}, r\right)\right]\right\}$ by standard tomographic reconstruction techniques, e.g., filtered backprojection. ${ }^{6}$ It is shown in Fig. 3 (left) as a function of position on the virtual section through the sample and is homogeneous throughout the sample $\left[\mu(x, y)=2.4 \pm 0.1 \mathrm{~cm}^{-1}\right]$, corresponding to a homogeneous polyethylene density of $\rho=0.88 \pm 0.04 \mathrm{~g} / \mathrm{cm}^{3}$.

The reconstruction of the SAXS data is more involved. Within first Born approximation, the SAXS pattern at a given position in the tomographic scan is given by [cf. Fig. 1(b)]
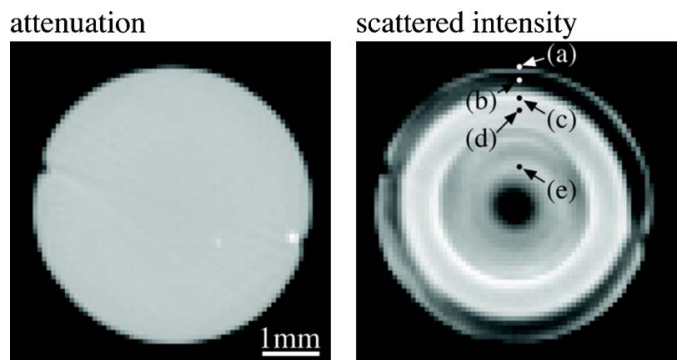

FIG. 3. Reconstructed attenuation coefficient $\mu$ and integral scattered intensity along the $q_{z}$ axis $\left(q_{r}=0\right)$ as a function of $x$ and $y$ on a virtual section through the sample. The diffraction patterns reconstructed at the locations marked in the right image are shown in Fig. 4. marked in the right image are shown in Fig. 4.
Downloaded 19 Mar 2007 to 131.169 .95 .147 . Redistribution subject to ÁlP lice

$$
I_{\mathbf{q}}(r, \phi)=I_{0} \int d s f(\phi, s, r) p_{\mathbf{q}, \phi}(x, y) g(\phi, s, r),
$$

where $I_{\mathbf{q}}(r, \phi)$ is the full SAXS pattern measured at point $(r, \phi)$ as a function of $\mathbf{q}=\left(q_{r}, q_{z}\right), p_{\mathbf{q}, \phi}(x, y)$ is the SAXS cross section at the location $(x, y)$ under illumination from the angle $\phi$, and $f(\phi, s, r)=\exp \left\{-\int_{-\infty}^{s} d s^{\prime} \mu\left[x\left(s^{\prime}, r\right), y\left(s^{\prime}, r\right)\right]\right\}$ and $g(\phi, s, r)=\exp \left\{-\int_{s}^{\infty} d s^{\prime} \mu\left[x\left(s^{\prime}, r\right), y\left(s^{\prime}, r\right)\right]\right\}$ describe the attenuation of the incident and the forward scattered beam, respectively. $f$ and $g$ can be combined and pulled out of the integral in Eq. (1). At each position of the tomographic scan

$$
I_{\mathbf{q}}(r, \phi)=I_{1} \int d s p_{\mathbf{q}, \phi}[x(s, r), y(s, r)],
$$

where $I_{1}$ is the transmitted intensity of the sample. Thus, to eliminate the effect of attenuation inside the sample, the measured SAXS patterns need to be normalized by the transmitted intensity $I_{1}$. In general, $p_{\mathbf{q}, \phi}(x, y)$ is different and uncorrelated for each angle $\phi$ and $q_{r} \neq 0$, such that Eq. (2) cannot be inverted without additional knowledge about the sample. The cross sections along the $q_{z}$ direction $\left(q_{r}=0\right)$, however, are independent of the angle $\phi$ and can be reconstructed using standard tomographic reconstruction techniques. If additional symmetry is present in the sample, e.g., isotropy or fiber symmetry along the $q_{z}$ direction, the $p_{\mathbf{q}, \phi}(x, y)$ are independent of $\phi$ as well and the full threedimensional reciprocal space information can be reconstructed around each location in the sample.

We have reconstructed the local small-angle scattering cross section of the polyethylene rod in the $q_{z}$ direction. The integral of the scattering signal along the $q_{z}$ axis is shown on the right in Fig. 3. While in absorption contrast the sample appeared homogeneous, this is not the case for the scattering signal that clearly shows the core-shell structure of the rod. At each location in the sample, the full SAXS pattern along the $q_{z}$ direction is available from the tomographic reconstruction. Figure 4 (left) shows these SAXS patterns at different locations inside the rod referred to in Fig. 3.

At the periphery of the rod, an almost latticelike arrangement of alternating crystalline and amorphous lamellae is indicated by strong and clear diffraction peaks. In Figs. 4(a) and 4(b) they are marked by arrows. In the bright shell in Fig. 3 the structure is dominated by the more or less diffuse scattering of uncorrelated domains [cf. Figs. 4(c) and 4(d)] (stacked crystalline and amorphous lamellae of varying thickness ${ }^{7}$ ). Towards the center [cf. Fig. 4(e)] discrete scattering is observed, again. The corresponding broad long period reflection indicates some short-range correlation among neighboring lamellae. Nevertheless, the homogeneity of layer thicknesses and distances remains much smaller than that observed at the periphery of the rod. These observations can be explained by the variations of both the temperature and the shear program during material processing as a function of the radial coordinate in the rod. Since the polyethylene rod is strongly fiber textured along its cylinder axis that was aligned with the rotation axis in the tomographic scan (cf. Fig. 2), the full reciprocal space at each location in the sample can be reconstructed. Figure 4 (right) shows sections through reciprocal space at the locations marked in Fig. 3. The diffraction patterns are rotationally symmetric about the

o AlP license or copyright, see http://apl.aip.org/apl/copyright.jsp 


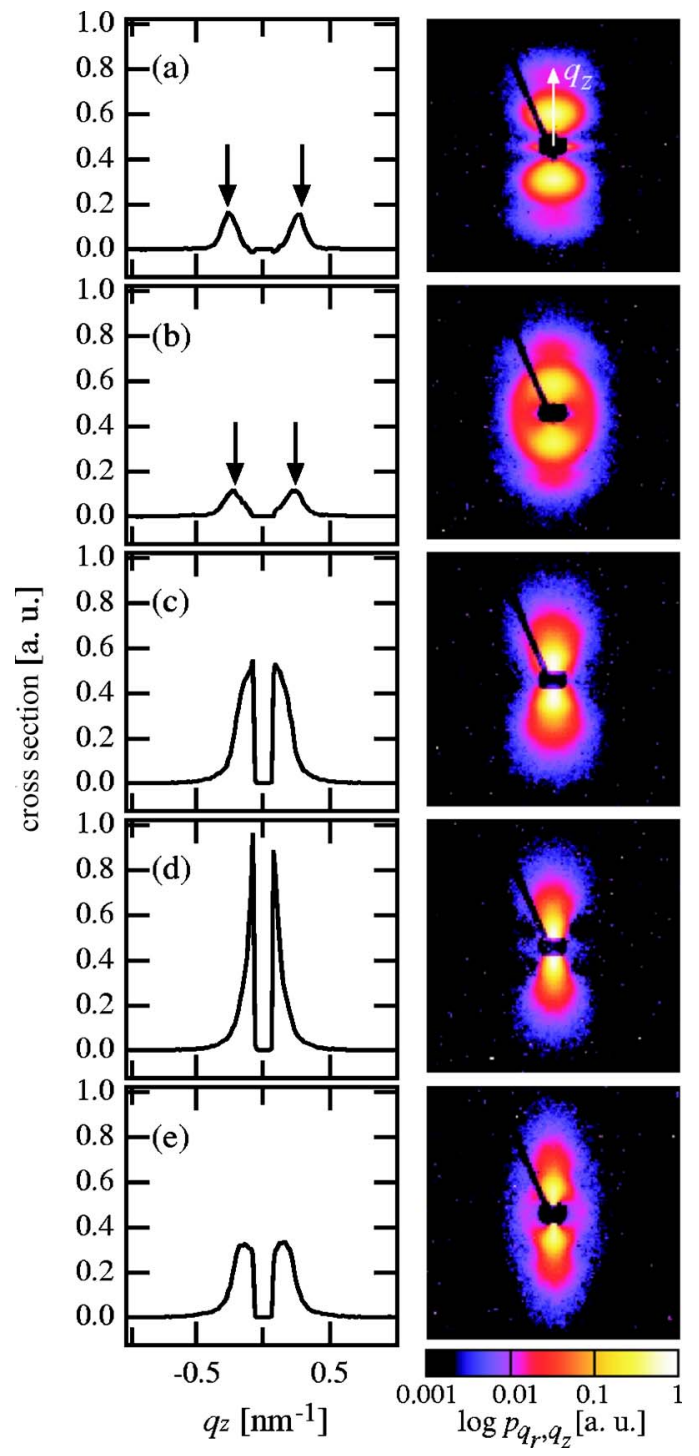

FIG. 4. (Color online) Reconstructed SAXS cross section along the $q_{z}$ direction $\left[p_{\left(0, q_{z}\right)}(x, y)\right.$, left $]$ and $2 \mathrm{D}$ sections through reciprocal space $\left[p_{\left(q_{v}, q_{7}\right)}(x, y)\right.$, right $]$ at different locations $(x, y)$ in the tomographic reconstruction shown in Fig. 3. The arrows indicate the period of the lamellar structure.

SAXS microtomography as an invasive, but nondestructive technique allows one to gain information about the local nanostructure inside a specimen, for example, with fiber texture. In the example of the polyethylene rod, the constant absorption shows that density and thus crystallinity are almost constant. Nevertheless, considerable variation of the semicrystalline structure with several zones is documented along the radial coordinate in the cross section by means of SAXS tomography. For comparison, conventional SAXS from sliced samples using a larger beam can only resolve a binary core-shell structure ${ }^{4}$ and requires destructive sample preparation.

SAXS tomography can be easily combined with other scanning tomography techniques, such as fluorescence microtomography. Micro-SAXS experiments like this one require a high flux in a small and well collimated beam. Higher spatial and angular resolution can be obtained at third generation undulator sources that are optimized for brilliance, i.e., flux per source size, divergence, and energy bandpass. At higher spatial resolution, the radiation dose will increase significantly. Although no radiation damage was observed in the given example, radiation damage issues will become more and more relevant as the spatial resolution is increased.

The authors thank R. Döhrmann and M. Dommach for their excellent support during the experiments carried out at beamline BW4. The development of refractive x-ray lenses is supported by the German Ministry of Education and Research (BMBF) under Grant No. 05KS4PA1/9.

${ }^{1}$ A. S. Simionovici, M. Chukalina, C. Schroer, M. Drakopoulos, A. Snigirev, I. Snigireva, B. Lengeler, K. Janssens, and F. Adams, IEEE Trans. Nucl. Sci. 47, 2736 (2000); C. G. Schroer, J. Tümmler, T. F. Günzler, B. Lengeler, W. H. Schröder, A. J. Kuhn, A. S. Simionovici, A. Snigirev, and I. Snigireva, Proc. SPIE 4142, 287 (2000); C. G. Schroer, Appl. Phys. Lett. 79, 1912 (2001); L. Vincze, B. Vekemans, I. Szalóki, K. Janssens, R. Van Grieken, H. Feng, K. W. Jones, and F. Adams, Proc. SPIE 4503, 240 (2002); B. Golosio, A. Simionovici, A. Somogyi, L. Lamelle, M. Chukalina, and A. Brunetti, J. Appl. Phys. 94, 145 (2003); B. Golosio, A. Somogyi, A. Simionovici, P. Bleuet, J. Susini, and L. Lamelle, Appl. Phys. Lett. 84, 2199 (2004); D. H. McNear, Jr., E. Peltier, J. Everhart, R. L. Chaney, S. Sutton, M. Newville, M. Rivers, and D. L. Sparks, Environ. Sci. Technol. 39, 2210 (2005).

${ }^{2}$ C. G. Schroer, M. Kuhlmann, T. F. Günzler, B. Lengeler, M. Richwin, B. Griesebock, D. Lützenkirchen-Hecht, R. Frahm, E. Ziegler, A. Mashayekhi, D. Haeffner, J.-D. Grunwaldt, and A. Baiker, Appl. Phys. Lett. 82, 3360 (2003).

${ }^{3}$ C. Riekel, Rep. Prog. Phys. 63, 233 (2000); S. V. Roth, M. Burghammer, C. Riekel, P. Müller-Buschbaum, A. Diethert, P. Panagiotou, and H. Walter, Appl. Phys. Lett. 82, 1935 (2003).

${ }^{4}$ N. Stribeck, A. Almendarez-Camarillo, S. Cunis, R. K. Bayer, and R. Gehrke, Macromol. Chem. Phys. 205, 1445 (2004).

${ }^{5}$ B. Lengeler, C. G. Schroer, M. Kuhlmann, B. Benner, T. F. Günzler, O. Kurapova, F. Zontone, A. Snigirev, and I. Snigireva, J. Phys. D 38, A218 (2005).

${ }^{6}$ A. C. Kak and M. Slaney, Principles of Computerized Tomographic Imaging (IEEE, New York, 1988).

${ }^{7}$ N. Stribeck, Macromol. Chem. Phys. 205, 1455 (2004); N. Stribeck, A. Almendarez-Camarillo, and R. K. Bayer, ibid. 205, 1463 (2004). 